\title{
Molecular characterization of Plasmodium juxtanucleare in Thai native fowls based on partial cytochrome $C$ oxidase subunit I gene
}

\author{
Tawatchai Pohuang ${ }^{1,2}$, Sucheeva Junnu ${ }^{1,2, *}$ \\ ${ }^{1}$ Department of Veterinary Medicine, Faculty of Veterinary Medicine, Khon Kaen University, Khon Kaen 40002, Thailand \\ ${ }^{2}$ Research Group for Animal Health Technology, Faculty of Veterinary Medicine, Khon Kaen University,
}

Khon Kaen 40002, Thailand

\begin{abstract}
Avian malaria is one of the most important general blood parasites of poultry in Southeast Asia. Plasmodium (P.) juxtanucleare causes avian malaria in wild and domestic fowl. This study aimed to identify and characterize the Plasmodium species infecting in Thai native fowl. Blood samples were collected for microscopic examination, followed by detection of the Plasmodium cox I gene by using PCR. Five of the 10 sampled fowl had the desired 588 base pair amplicons. Sequence analysis of the five amplicons indicated that the nucleotide and amino acid sequences were homologous to each other and were closely related $(100 \%$ identity) to a P. juxtanucleare strain isolated in Japan (AB250415). Furthermore, the phylogenetic tree of the cox I gene showed that the P. juxtanucleare in this study were grouped together and clustered with the Japan strain. The presence of $P$. juxtanucleare described in this study is the first report of $P$. juxtanucleare in the Thai native fowl of Thailand.
\end{abstract}

Keywords: fowl, cytochrome C oxidase subunit I, Plasmodium juxtanucleare

\section{*Corresponding author}

Sucheeva Junnu

Department of Veterinary Medicine,

Faculty of Veterinary Medicine, Khon

Kaen University, 123, Mittraphap Frontage

Rd., Khon Kaen 40002, Thailand

Tel: +82-66-86236-5459

Fax: +82-66-43-202-404

E-mail: sucheeva@kku.ac.th

\section{ORCID}

Sucheeva Junnu

https://orcid.org/0000-0003-1081-7279

Tawatchai Pohuang

https://orcid.org/0000-0002-4634-3995

Conflict of Interest

The authors declare no conflicts of interest.

Received: March 11, 2019

Revised: May 27, 2019

Accepted: June 11, 2019

\section{Introduction}

Avian malaria, caused by Plasmodium spp., is an important blood parasite disease of poultry because it results in poor meat quality and egg production [1]. The clinical signs of avian malaria in host birds vary from no apparent signs to severe anemia and death, and the mortality rate associated with avian malaria has been reported to be as high as $80 \%-90 \%$ [2]. Plasmodium spp. have been reported to infect in various domestic fowl; Plasmodium (P.) gallinaceum occurs in jungle fowl and domestic fowl, while $P$. juxtanucleare has been detected in domestic fowl and turkeys. Avian malaria is common in tropical countries such as Asia, East and South Africa, and Central and South America [3]. P. juxtanucleare was first described in Brazil [4] and has since been reported in Asian countries such as Malaysia [5], Vietnam [6], and Thailand [7].

Generally, detection of Plasmodium in infected birds can be performed by Giemsa staining of a thin blood smear, which may reveal erythrocytes with trophozoites, merozoites, and/or gametocytes of Plasmodium spp. [8]. That method is performed by specialist workers familiar with detecting and identifying parasites in blood samples. In addition, a DNA-based detection method for identifying avian malaria parasites in blood samples has been reported [9]. The mitochondrial genome of apicomplexan parasite encodes three proteincoding genes (cytochrome b [cyt b], cytochrome c oxidase subunits I [cox I] and III [cox III]) and six fragments of large subunit rRNA genes. The cyt $b$ and cox I genes have been widely used in the molecular detection of avian malaria parasites such as $P$. gallinaceum $[10,11]$. The cox I gene is the target region used for species identification and phylogenetic analysis because it is the most highly conserved mitochondrially encoded protein [12-14]. In Thailand, a molecular characterization study of $P$. juxtanucleare reported only a partial sequence of the cyt $b$ gene of Burmese red jungle fowls [7]. To date, there are no cox I gene-based reports on P. juxtanucleare infection in Thai native fowl. Therefore, the aim of this study was to identify and characterize the malaria parasites infecting Thai native fowl by using molecular tech- 
niques based on the partial cox I gene.

\section{Materials and Methods}

\section{Blood sample collection}

In 2016, ten of 50 Thai native fowl that had been raised in a backyard in northeast Thailand exhibited paleness and emaciation. These 10 fowl were suspected to be exhibiting subclinical signs of a blood parasite infection. Thus, blood samples were collected and submitted to the Veterinary Diagnostic Laboratory, Faculty of Veterinary Medicine, Khon Kaen University, Thailand.

\section{Blood smear analysis}

The collected blood smears were prepared as thin blood films and fixed with absolute methanol for $10 \mathrm{~min}$, left to dry, and then stained with $10 \%$ Giemsa solution for $30 \mathrm{~min}$. The stained slides were then washed with tap water and airdried. Each slide was examined under a light microscope (Olympus, Tokyo, Japan) using 1,000× magnification with oil immersion. Avian malaria parasites were observed and identified as described by Valkiunas [11].

\section{DNA extraction and polymerase chain reaction (PCR)}

The DNA of each of the 10 malaria-suspected fowl was extracted from each blood sample by using a TIANamp Blood DNA Maxi Kit (TIANGEN Biotech, Beijing, China) following the manufacturer's instruction. The PCR method described by Hellgren et al. [15] for detection of the cox I gene of Plasmodium spp. was used. A partial cox I gene with a length of 588 base pairs (bp) was amplified by using forward primer CoxI-F1 (5'-GCGTACTTTGGACCGAAAAA-
3') and reverse primer CoxI-R1 (5'- CATCCAGTACCACCACCAAA -3') as described in a previous study of cox I genes of $P$. gallinaceum in Thailand [9]. The PCR was performed in a DNA thermocycler (Major cycler, Major Science, Taiwan) using a DreamTaq Green PCR Master Mix $(2 \times)$ (ThermoFisher Scientific, USA) following the manufacturer's instruction. Briefly, $3 \mu \mathrm{L}$ of each DNA sample was added to $22 \mu \mathrm{L}$ of the PCR mixture, which was composed of $12.5 \mu \mathrm{L}$ of DreamTaq Green PCR Master Mix solution, $0.5 \mu \mathrm{L}$ of $10 \mu \mathrm{M}$ of each forward and reverse primer, and $8.5 \mu \mathrm{L}$ of nuclease-free water. Thermal cycling was carried out under the optimized conditions of $95^{\circ} \mathrm{C}$ for $5 \mathrm{~min}$, followed by 40 cycles of $95^{\circ} \mathrm{C}$ for $30 \mathrm{sec}, 55^{\circ} \mathrm{C}$ for $30 \mathrm{sec}$, and $68^{\circ} \mathrm{C}$ for $60 \mathrm{sec}$, followed by a final period at $68^{\circ} \mathrm{C}$ for 10 min. The PCR products were analyzed by electrophoresis using $1.2 \%$ agarose gels containing $1 \times$ of GelRed ${ }^{\mathrm{TM}}$ Nucleic Acid Gel Stain. Each gel was electrophoresed at 100 volts for $30 \mathrm{~min}$ and then visualized by using ultraviolet light in the Gel Doc ${ }^{\mathrm{TM}}$ XR+ Gel Documentation System (BioRad, USA).

\section{Nucleotide sequence analysis of the cox I gene}

The PCR products were purified for sequencing by using a NucleoSpin Gel and PCR Clean-up (Macherey-Nagel, Germany) kit following the manufacturer's instructions. Sequencing was performed by First BASE Laboratories Sdn Bhd, Malaysia). The obtained nucleotide sequences were analyzed to determine sequence identities by using the BLASTN program (https://www.ncbi.nlm.nih.gov/BLAST) and were assembled by using BioEdit software version 7.1.11 [16]. The BankIt tool was used for analysis of the sequence data of the 588 bp fragment submissions in the GenBank database (https:/www.ncbi.nlm.nih.gov/WebSub/?tool=genbank). The

Table 1. GenBank accession numbers of reference strains of Plasmodium species and Leucocytozoon species, country of origin used in neighbor-joining analysis of cox I sequences

\begin{tabular}{|c|c|c|c|}
\hline Plasmodium species & Host species & Geographic origin & GenBank accession number \\
\hline P. juxtanucleare -KKUTH02 & Chicken & Thailand & KY930301 \\
\hline P. juxtanucleare -KKUTH03 & Chicken & Thailand & KY930302 \\
\hline P. juxtanucleare -KKUTH04 & Chicken & Thailand & KY930303 \\
\hline P. juxtanucleare -KKUTH07 & Chicken & Thailand & KY930304 \\
\hline P. juxtanucleare -KKUTH10 & Chicken & Thailand & KY930305 \\
\hline P. juxtanucleare & n.a & Japan & AB250415 \\
\hline P. gallinaceum & n.a & Philippine & $\mathrm{AB} 250690$ \\
\hline P. gallinaceum & n.a & Thailand & KP025674 \\
\hline P. gallinaceum & n.a & Japan & AB599930 \\
\hline P. relictum & n.a & United Kingdom & LN835311 \\
\hline P. relictum & Spheniscus demersus & South Africa & KY653774 \\
\hline P. circumflexum & Troglodytes troglodytes & Lithuania & KY653762 \\
\hline P. elongatum & Spheniscus demersus & South Africa & KY653802 \\
\hline Leucocytozoon caulleryi & n.a & Japan & AB302215 \\
\hline Leucocytozoon sabrazesi & n.a & Malaysia & AB299369 \\
\hline
\end{tabular}

P. juxtanucleare, Plasmodium juxtanucleare; n.a, not available; P. gallinaceum, Plasmodium gallinaceum; P. relictum, Plasmodium relictum; P. circumflexum, Plasmodium circumflexum; P. elongatum, Paramonostomum elongatum. 
sequences of KKUTH02, KKUTH03, KKUTH04, KKUTH07, and KKUTH10 have been deposited in GenBank databases with the accession numbers KY930901, KY930902, KY930903, KY930904, and KY930905, respectively.

\section{Phylogenetic analysis}

The identified partial cox I gene sequences were analyzed by comparison with other Plasmodium spp. sequences obtained from the GenBank database. GenBank accession numbers for the mitochondrial sequences (Table 1) were used during the analysis. The nucleotide and deduced amino acid sequence identities and multiple sequence alignment analyses were analyzed by using ClustalW of BioEdit software [17]. Distance analyses of and phylogenetic tree generation from the nucleotide sequences were performed by applying the neighbor-joining and maximum likelihood methods by using MEGA6 software version 6.06 [18] based on the Kimura 2parameter model with 1,000 replications of bootstrap values. Leucocytozoon caulleryi and Leucocytozoon sabrazesi sequences were used as out-of-group members to root the tree.

\section{Results}

\section{Microscopic examination}

Examination of the Giemsa-stained blood thin smears revealed that none of the ten Thai native fowl sampled had visible plasmodial stages in the blood smears.

\section{Homology analysis}

Agarose gel electrophoresis of five of the ten Thai native fowl samples revealed the presence of $588 \mathrm{bp}$ amplicons (Fig. 1). The results of nucleotide sequence and amino acid analyses revealed $100 \%$ nucleotide identities of the ampli- cons to the cox I gene of $P$. juxtanucleare. The sequence alignment of the nucleotide and amino acid sequences (Figs. 2 and 3 ) of the five amplicons isolated in this study were $100 \%$ similar to each other and were closely related to the P. juxtanucleare Japan strain (AB250415). The amino acid sequence identity similarities among samples KKUTH02, KKUTH03, KKUTH04, KKUTH07, KKUTH10, and the Japan strain were $100 \%$.

\section{Phylogenetic analysis}

The phylogenetic tree of the partial cox I gene of P. juxtanucleare based on including 17 sequences of the GenBank

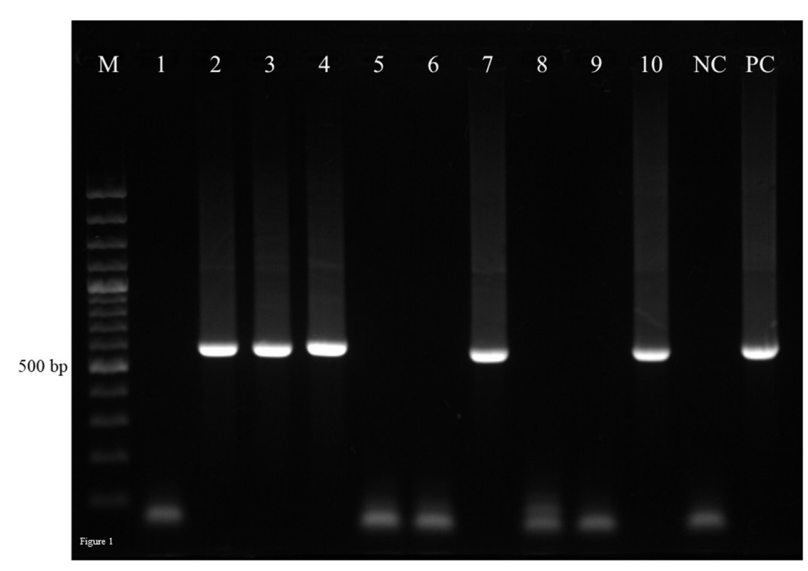

Fig. 1. PCR Electrophoresis gel demonstrating P. juxtanucleare cox I gene amplification with CoxI-F1 and CoxI-R1. M. 100 bp DNA marker ladder, Lanes: 1. KKUTH01; 2. KKUTH02; 3. KKUTH03; 4. KKUTH04; 5. KKUTH05; 6. KKUTH06; 7. KKUTH07; 8. KKUTH08; 9. KKUTH09; 10. KKUTH10.

$\mathrm{NC}$, negative control; PC, positive control.

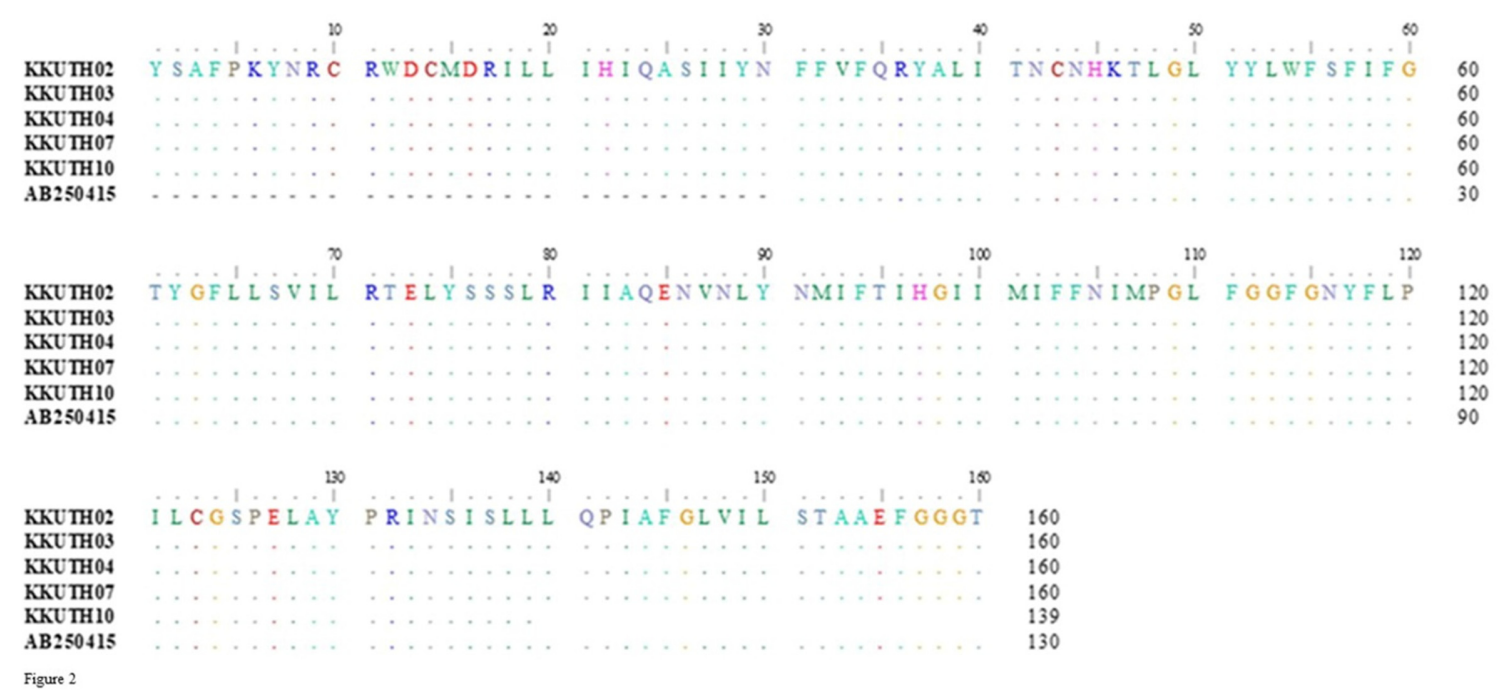

Fig. 2. Deduced amino acid sequences alignment of $P$. juxtanucleare cox I gene from Thai native fowls compared with reference sequence. Dots in the sequence signify the similarity of the amino acids. These sequences of $P$. juxtanucleare Thai native chicken isolates (KKUTH02, KKUTH03, KKUTH04, KKUTH07 and KKUTH10) were identical to that of P. juxtanucleare [NCBI reference number: AB250415]. Numbers indicate the amino acid positions. 


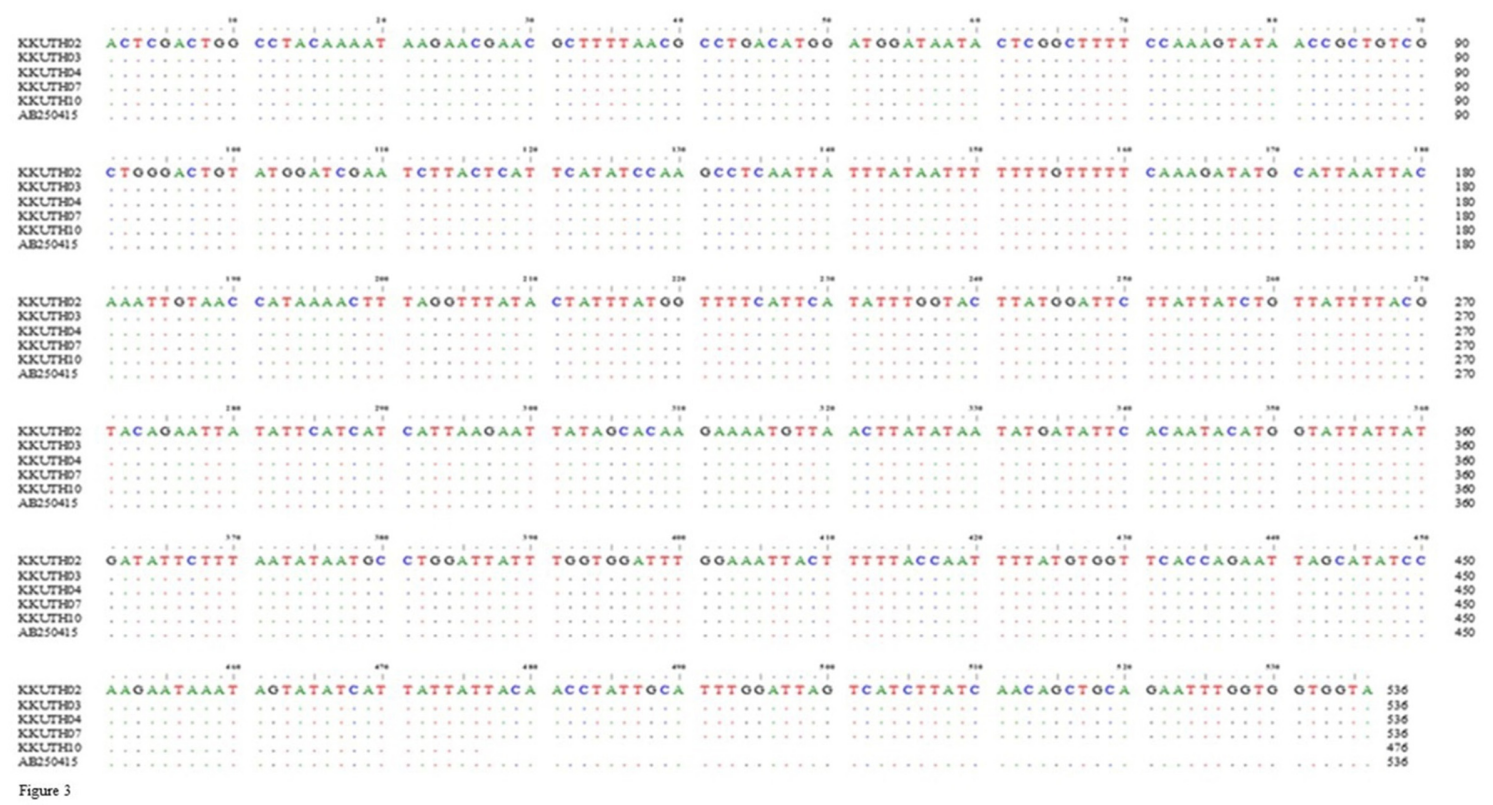

Fig. 3. Nucleotide sequence of Thai isolates of $P$. juxtanucleare cox I gene. Dots in the sequence signify the similarity of the nucleotides. Nucleotide sequences of $P$. juxtanucleare Thai isolates (KKUTH02, KKUTH03, KKUTH04, KKUTH07 and KKUTH10) were identical to that of P. juxtanucleare [NCBI reference number: AB250415]. Numbers indicate the nucleotide positions.

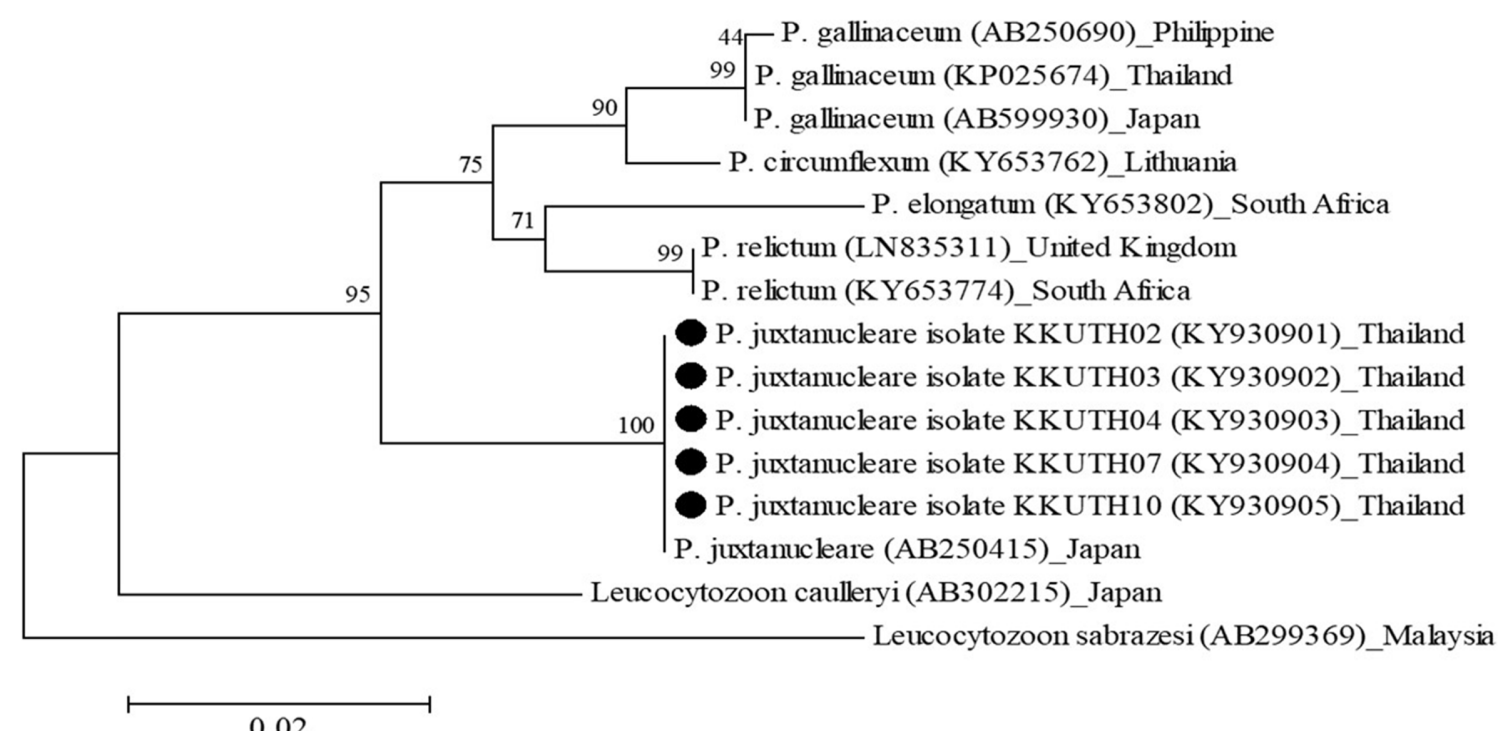

Fig. 4. The phylogenetic tree of the partial cox I gene sequences of Plasmodium species. This tree was generated using the neighborjoining method. Bootstrap values were obtained from 1,000 replications. The scale bar represents the number of substitutions per site. KKUTH02, KKUTH03, KKUTH04, KKUTH07 and KKUTH10 were received from the five positive samples in Thai native folws. Other sequences were acquired from GenBank (Table 1), and Leucocytozoon caulleryi and Leucocytozoon sabrazesi were used as outgroup.

reference strains formed two major groups. P. juxtanuclearepositive samples KKUTH02, KKUTH03, KKUTH04, KKUTH07, and KKUTH10 were closely related (100\% similarity), and they also had $100 \%$ similarity with the P. juxtanucleare Japan strain. The five sequences obtained in this study were separated from other Plasmodium spp. and from the Leucocytozoon species outlier (Fig. 4). They had 95.7\%,
95.7\%, and $95.5 \%$ similarities with $P$. gallinaceum strains from Thailand, Japan, and Philippines, respectively. The greatest phylogenetic distance between our sequences and other published strains were for the partial cox I genes of $P$. elongatum, P. circumflexum, and P. relictum $(95.1 \%, 95.7 \%$, and 96.1\% similarities, respectively) from South Africa, Lithuania, United Kingdom, and USA. The results showed that $P$. 
juxtanucleare in Thailand are genetically related to P. juxtanucleare of Asian strain.

\section{Discussion}

The aim of this study was to identify the presence of $P$. juxtanucleare in Thai native fowls and to compare the partial cox I sequences obtained from our samples with those of other Plasmodium spp. isolated around the world. Included in our analyses were various GenBank sequences including one each from wren (Troglodytes troglodytes) and black-foot penguin (Spheniscus demersus) [19] and that for P. gallinaceum isolated from Gallus gallus domesticus in Japan [20]. Also included were the PCR amplicons and the percentage identity from the BLASTN software of the five positive field samples. The phylogenetic tree developed for the cox I gene revealed that the five isolates were grouped together and were most similar to the Japan strain of $P$. juxtanucleare. Similarly, the nucleotide and amino acid identities of the five samples (KKUTH02, KKUTH03, KKUTH04, KKUTH07 and KKUTH10) showed a close relationship with the $P$. juxtanucleare isolated in Japan (100\% similarity).

Avian malaria is well recognized in poultry, but only a few studies on its molecular characterization have been reported. $P$. juxtanucleare is one of the most common species of Plasmodium to cause malaria in poultry. $P$. juxtanucleare has mainly been detected in quail, turkey, and domestic fowls $[11,21]$ in several countries. Several Plasmodium spp. cannot be identified based solely on blood stage morphology via microscopic examination, thus molecular technology has to be used for accurate species identification. Moreover, microscopy-based detection of avian malaria presence is less sensitive than the molecular-based method and such sensitivity can be further reduced by low quality blood sample slides or by the inexperience of the investigator. As shown in the present study, PCR assay amplification of the cox I gene fragment can detect $P$. juxtanucleare from fowl blood samples even though the parasite was not detected by light microscopy of blood smears. Therefore, the sensitivity of the PCR assay is higher than that of direct microscopic examination. This finding suggests that PCR assays would be a suitable method for screening fowl for the presence of P. juxtanucleare infection.

Plasmodium genes were detected in blood samples by using PCR. A specific PCR fragment of the predicted size (588 bp) was obtained. The partial cox I genes of Plasmodium from the sampled Thai native fowl were sequenced and neighbor-joining analysis placed the partial cox I genes into a cluster with a $P$. juxtanucleare isolated from Japan [20]. The finding of $P$. juxtanucleare infection in this study was similar to that in a previous study in Thailand [7]. However, those authors reported molecular characterization of the cyt $b$ gene in infected Burmese red jungle fowls (Gallus gallus spadiceus), whereas, our work characterized them based on the cox I gene in Thai native fowl.
A limitation of this study is the small amount of data available in the DNA database for use in comparing strains of the parasite; regardless, our molecular analysis indicated that the parasite isolated is an Asian Plasmodium strain. More molecular data of $P$. juxtanucleare and its natural reservoir are required to further describe the evolution and distribution of Plasmodium. Herein, we provide the first nucleotide sequence diversity of the cox I gene of $P$. juxtanucleare from field isolates in Thailand. The partial cox I gene analysis sequencing revealed that the $P$. juxtanucleare detected from native fowl in Thailand were very similar to $P$. juxtanucleare isolated in Japan [20]. This study shows the importance of using molecular assays to screen for parasite presence as the fowl in our study had subclinical signs and would have been misdiagnosed based on the microscopic finding. Currently, nucleotide sequence analysis is the only molecular assay that is useful for differentiating between $P$. gallinaceum and $P$. juxtanucleare. The restriction fragment length polymorphism assay was developed for species identification and could be useful for disease diagnosis, but it lacks a DNA sequencing ability. Future studies should investigate samples from commercial poultry that are reared in an area close to backyard birds. Such study will help determine the presence and genetic diversity of Plasmodium parasites in commercial farms of Thailand and will assist in monitoring Plasmodium spp. presence.

In conclusion, our results provide important information on the detection and phylogenetic relationships of P. juxtanucleare present in Thai native fowl in Thailand. The results showed a close relationship among the five isolates and an isolate from Japan. Additionally, the results indicate that the use of PCR-based blood assays is suitable for diagnosis and screening of the P. juxtanucleare status in poultry.

\section{Acknowledgments}

This research was financially supported by the Research Fund of Faculty of Veterinary Medicine and Research Group for Animal Health Technology, Faculty of Veterinary Medicine, Khon Kaen University, Khon Kaen, Thailand.

\section{References}

1. Ruff MD. Important parasites in poultry production systems. Vet Parasitol 1999;84:337-347.

2. Williams RB. Avian malaria: clinical and chemical pathology of Plasmodium gallinaceum in the domesticated fowl Gallus gallus. Avian Pathol 2005;34:29-47.

3. Taylor MA, Coop RL, Wall RL. Parasites of Poultry and Gamebirds. In: Taylor MA, Coop RL, Wall RL (eds.). Veterinary Parasitology. 3rd ed. pp. 459-557, Blackwell Publishing Ltd., Oxford, 2007.

4. Versiani V, Gome BF. Sobre um novo hematozoario da galinha - "Plasmodium juxtanucleare n. sp. (Nota previa). Rev Bras Biol 1941;1:231-233.

5. Fernando MA, Dissanaike AS. Studies on Plasmodium 
gallinaceum and Plasmodium juxtanucleare from the Malayan jungle fowl Gallue gallus spadiceus. Southeast Asian J Trop Med Public Health 1975;6:25-32.

6. Kissinger JC, Souza PC, Soarest CO, Paul R, Wahl AM, Rathore D, McCutchan TF, Krettli AU. Molecular phylogenetic analysis of the avian malarial parasite Plasmodium (Novyella) juxtanucleare. J Parasitol 2002;88:769-773.

7. Tattiyapong M, Deemagarn T, Mohkeaw K. Molecular characterization of Plasmodium juxtanucleare in Burmese red junglefowls (Gallus gallus spadiceus) in Thailand. J Protozool Res 2016;26:1-10.

8. Atkinson CT. Avian Malaria. In: Atkinson CT, Thomas NJ, Hunter DB (eds.). Parasitic Diseases of Wild Birds. pp. 3553, Wiley-Blackwell Publishing, Iowa, 2008.

9. Pattaradilokrat S, Tiyamanee W, Simpalipan P, Kaewthamasorn M, Saiwichai T, Li J, Harnyuttanakorn P. Molecular detection of the avian malaria parasite Plasmodium gallinaceum in Thailand. Vet Parasitol 2015;210:1-9.

10. Joseph JT, Aldritt SM, Unnasch T, Puijalon O, Wirth DF. Characterization of a conserved extrachromosomal element isolated from the avian malarial parasite Plasmodium gallinaceum. Mol Cell Biol 1989;9:3621-3629.

11. Valkiūnas G, Iezhova TA, Loiseau C, Smith TB, Sehgal RN. New malaria parasites of the subgenus Novyella in African rainforest birds, with remarks on their high prevalence, classification and diagnostics. Parasitol Res 2009;104:1061-1077.

12. Ekala MT, Khim N, Legrand E, Randrianarivelojosia M, Jambou R, Fandeur T, Menard D, Assi SB, Henry MC, Rogier C, Bouchier C, Mercereau-Puijalon O. Sequence analysis of Plasmodium falciparum cytochrome b in multiple geographic sites. Malar J 2007;6:164.

13. Perkins SL. Molecular systematics of the three mitochondrial protein-coding genes of malaria parasites: corroborative and new evidence for the origins of human malaria. Mitochondrial DNA 2008;19:471-478.

14. Farrugia C, Cabaret O, Botterel F, Bories C, Foulet F, Costa $\mathrm{JM}$, Bretagne S. Cytochrome $\mathrm{b}$ gene quantitative PCR for diagnosing Plasmodium falciparum infection in travelers. J Clin Microbiol 2011;49:2191-2195.

15. Hellgren O, Waldenström J, Bensch S. A new PCR assay for simultaneous studies of Leucocytozoon, Plasmodium, and Haemoproteus from avian blood. J Parasitol 2004;90:797-802.

16. Hall TA. BioEdit: a user-friendly biological sequence alignment editor and analysis program for Windows 95/98/NT. Nucleic Acids Symp Ser 1999;41:95-98.

17. Thompson JD, Higgins DG, Gibson TJ. CLUSTAL W: improving the sensitivity of progressive multiple sequence alignment through sequence weighting, position-specific gap penalties and weight matrix choice. Nucleic Acids Res 1994;22:46734680 .

18. Tamura K, Stecher G, Peterson D, Filipski A, Kumar S. MEGA6: molecular evolutionary genetics analysis version 6.0. Mol Biol Evol 2013;30:2725-2729.

19. Murata K, Nii R, Sasaki E, Ishikawa S, Sato Y, Sawabe K, Tsuda Y, Matsumoto R, Suda A, Ueda M. Plasmodium (Bennettinia) juxtanucleare infection in a captive white earedpheasant (Crossoptilon crossoptilon) at a Japanese zoo. J Vet Med Sci 2008;70:203-205.

20. Omori S, Sato Y, Isobe T, Yukawa M, Murata K. Complete nucleotide sequences of the mitochondrial genomes of two avian malaria protozoa, Plasmodium gallinaceum and Plasmodium juxtanucleare. Parasitol Res 2007;100:661-664.

21. Bennett GF, Bishop MA, Peirce MA. Checklist of the avian species of Plasmodium Marchiafava and Celli, 1885 (Apicomplexa) and their distribution by avian family and Wallacean life zones. Syst Parasitol 1993;26:171-179. 